\title{
Evidências cientificas da prática da violência contra a pessoa idosa: revisão integrativa
}

Scientific evidence of the violence against the older adult: an integrative review Evidencias científicas de la práctica de violencia contra personas mayores: revisión integradora

Maria de Nazaré de Souza Ribeiro' ${ }^{1}$ io hitps://orcid.org/0000-0002-7641-1004

Fátima Helena do Espírito Santo ${ }^{2}$ io https://orcid.org/0000-0003-4611-5586,

Cleisiane Xavier Diniz ${ }^{1}$ io https://orid.org/0000-0003-4689-6204

Karla Brandão de Araújjo ${ }^{1}$ io https:/orcid.org/0000-0002-0563-9562

Maressa Gasparoto Lenglube Lisboa' io https://orcid.org/0000-0002-3308-5990

Cássia Rozária da Silva Souza ${ }^{1}$ io hitps://orcid.org/0000-0001-9790-3713

\section{Como citar:}

Ribeiro MN, Santo FH, Diniz CX, Araújo KB, Lisboa MG, Souza CR. Evidências científicas da prática da violência contra a pessoa idosa: revisão integrativa. Acta Paul Enferm. 2021;34:eAPE00403.

DOI

http://dx.doi.org/10.37689/actaape/2021AR00403

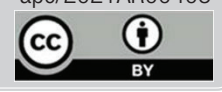

Descritores

Violência; Idoso; Violência doméstica; Exposição à violência; Idoso fragilizado

Keywords

Violence; Aged; Domestic violence; Exposure to violence; Frail elderly

Descriptores

Violencia; Anciano; Violencia doméstica; Exposición a violencia; Anciano frágil

Submetido
29 de Fevereiro de 2020
Aceito
20 de Agosto de 2020

Autor correspondente

Maria de Nazaré de Souza Ribeiro E-mail:mnribeiro2@gmail.com

\section{Resumo}

Objetivo: Analisar as evidências científicas da violência praticada contra a pessoa idosa, com destaque para a prevalência, o perfil da vítima e os fatores de risco.

Métodos: Revisão integrativa de artigos disponíveis nas bases de dados da PubMed®, Cumulative Index to Nursing and Allied Health Literature (CINAHL), Banco de Dados em Enfermagem (BDENF), Scientific Electronic Library Online (SciELO) e Literatura Latino-Americana e do Caribe em Ciências da Saúde (LILACS), publicados entre 2015 a 2019, a partir dos descritores: "idoso", "elderly", "older person", "aged", "old", "exposure to violence", "exposição à violência", "aging", envelhecimento", "elder abuse", "domestic violence", "violence", "violência", "maus-tratos ao idoso" e "violência doméstica". Para refinamento, foram utilizadas as quatro fases do diagrama de fluxo de seleção de artigos do PRISMA.

Resultados: Foram identificados 17 artigos que apresentaram como fatores de risco aumentados para a violência contra a pessoa idosa idade avançada, disfuncionalidade familiar, falta de acesso a direitos sociais e condições crônicas de incapacidade.

Conclusão: 0 estudo traz contribuições diretas para os profissionais e setores interessados no enfrentamento da violência contra a pessoa idosa, a qual possui alta prevalência na sociedade atual.

\section{Abstract}

Objective: To analyze the scientific evidence of violence against the older adult, with emphasis on the prevalence, the profile of the victim, and the risk factors.

Methods: An integrative review of articles available in the PubMed $®$ databases, Cumulative Index to Nursing and Allied Health Literature (CINAHL), Nursing Database (BDENF), Scientific Electronic Library Online (SciEL0) and Latin American and Caribbean Research on Health Sciences (LILACS), published between 2015 and 2019, using the descriptors: "idoso", "older adult", "older person", "aged", "old", "exposure to violence", "exposição à violência", "aging", envelhecimento", "elder abuse", "domestic violence", "violence", "violência", "maustratos ao idoso" and "violência doméstica". As to refine it, the four phases of the PRISMA study selection flow diagram were used.

Results: 17 articles were identified which presented as increased risk factors for violence against the older adult, family dysfunction, lack of access to social rights and chronic conditions of disability.

Conclusion: The study brings direct contributions to professionals and sectors interested in facing violence against the older adult, which has a high prevalence in today's society. 


\section{Resumen}

Objetivo: Analizar las evidencias científicas de la violencia practicada contra personas mayores, con énfasis en la prevalencia, el perfil de la víctima y los factores de riesgo.

Métodos: Revisión integradora de artículos disponibles en las bases de datos de PubMed®, Cumulative Index to Nursing and Allied Health Literature (CINAHL), Banco de Dados em Enfermagem (BDENF), Scientific Electronic Library Online (SciELO) y Literatura Latinoamericana y del Caribe en Ciencias de la Salud (LILACS), publicados entre 2015 y 2019, a partir de los descriptores: "anciano", "elderly", "older person", "aged", "old", "exposure to violence", "exposición a la violencia", "aging", "envejecimiento", "elder abuse", "domestic violence", "violence", "violencia”, "malos tratos al anciano" y "violencia doméstica”. Para refinar la búsqueda, se utilizaron las cuatro fases del diagrama de flujo de selección de artículos PRISMA.

Resultados: Se identificaron 17 artículos que presentaron los siguientes factores de riesgo aumentados en la violencia contra personas mayores: edad avanzada, disfuncionalidad familiar, falta de acceso a derechos sociales y condiciones crónicas de incapacidad.

Conclusión: El estudio contribuye directamente para los profesionales y sectores interesados en el enfrentamiento a la violencia contra personas mayores, que tiene una alta prevalencia en la sociedad actual.

\section{Introdução}

A mudança na estrutura etária em direção ao envelhecimento altera a relação de dependência física, psicológica, social e econômica da pessoa idosa e gera demandas específicas para as famílias e as políticas sociais. ${ }^{(1,2)}$ Nesse contexto, a violência contra a pessoa idosa emerge como grave e crescente problema em um país que caminha para ser o sexto com maior número de idosos no mundo. Esse problema ocorre de diferentes formas e, dependendo do contexto cultural em que está inserida, sua identificação torna-se difícil, com sérias consequências para indivíduos, famílias, comunidades e gestores. ${ }^{(3)}$

A World Health Organization (WHO) ${ }^{(4)}$ define violência contra pessoas idosas como ato único ou repetido, que se faz presente em um relacionamento de confiança e cause danos, sofrimento ou angústia para a pessoa idosa. Pode ser representada por violência financeira, física, psicológica, sexual, medicamentosa, emocional/social, abandono, negligência e autonegligência. ${ }^{(5,6)}$

Em 2005, instituiu-se, no Brasil, o Plano de Ação para o Enfrentamento da Violência Contra a Pessoa Idosa ${ }^{(7)}$ que, em 2020, completa 15 anos, estabelecendo estratégias de ação, prevenção e enfrentamento dessa violência. Contudo, nos dias atuais, observa-se que o problema é ainda maior e mais preocupante. Todos os dias, são informadas situaçôes de violência contra pessoas idosas, seja em seu domicílio, nas Instituiçóes de Longa Permanência ou no ambiente externo de uso dos idosos. Estatísticas anuais apontam elevada prevalência da violência, a maioria praticada por pessoas próximas ao idoso. ${ }^{(8)}$
Dados divulgados pelo Ministério dos Direitos Humanos revelam que, em 2017, ocorreram, no Brasil, 33.133 denúncias e 68.870 violaçôes contra pessoas. Nas denúncias de violaçóes, $76,84 \%$ envolveram negligência, $56,47 \%$ implicaram violência psicológica e $42,82 \%$, abuso financeiro e econômico. A maior parte dos casos ocorreu na casa da própria vítima. ${ }^{(8)}$

No Brasil, dados quanto à violência e aos maus-tratos a idosos são difíceis de estimar, pois as famílias costumam ocultar o fato, o idoso sente-se desprotegido para proceder à denúncia, e os profissionais de saúde raramente notificam os casos. ${ }^{(8)}$ Além disso, reconhece-se que a violência não é unívoca, mas constitui fenômeno sócio-histórico complexo, que ocorre no âmbito de diferentes lugares, épocas, meios e circunstâncias. Em diversas situações, ela é praticada nos planos econômicos, político, moral, psicológico e físico e na violação de direitos básicos da pessoa humana, necessitando de um olhar para além das ocorrências relatadas pela vítima e por sua família. Diante do exposto, questiona-se: quais as evidências científicas sobre o fenômeno da violência e seus fatores associados na população idosa?

O objetivo deste estudo foi analisar as evidências científicas da violência praticada contra a pessoa idosa, destacando a prevalência, o perfil da vítima e os fatores de risco.

\section{Métodos}

Trata-se de uma revisão integrativa, composta de seis etapas: construção da questão de pesquisa, seleção dos critérios de inclusão e exclusão com busca 
na literatura com protocolo validado, categorização dos estudos, avaliação dos estudos incluídos, interpretação dos resultados e apresentação da revisão. ${ }^{(9)}$

Para o desenvolvimento da questão de pesquisa e escolha dos descritores na busca das evidências sobre a temática, utilizou-se a estratégia $\mathrm{PICo}^{(10)}$ (Quadro 1).

Quadro 1. Estratégia PICo utilizada no estudo

\begin{tabular}{|l|l|}
\hline Acrônimo & Descritores \\
\hline P - População: pessoa idosa & Idoso, elderly, older person, aged e old \\
\hline $\begin{array}{l}\text { I - Intervenção/Exposição: violência } \\
\text { intrafamiliar }\end{array}$ & $\begin{array}{l}\text { Exposure to violence, exposição à violência, } \\
\text { aging, envelhecimento }\end{array}$ \\
\hline $\begin{array}{l}\text { Co- Desfecho/Resultados/Contexto: } \\
\text { maus-tratos, ocorrência de violência }\end{array}$ & $\begin{array}{l}\text { Elder abuse, domestic violence, violence, } \\
\text { violência, maus-tratos ao idoso, violência } \\
\text { doméstica }\end{array}$ \\
\hline
\end{tabular}

Fonte: Polit DF, Beck CT. Fundamentos da pesquisa em enfermagem: avaliação de evidências para a prática de enfermagem. $9^{\mathrm{a}}$ ed. Porto Alegre: ArtMed; 2018. ${ }^{(10)}$

A pesquisa foi realizada de setembro de 2019 a janeiro de 2020, nas plataformas: PubMed ${ }^{\oplus}$, Cumulative Index to Nursing and Allied Health Literature (CINAHL), Banco de Dados em Enfermagem (BDENF), Scientific Electronic Library Online (SciELO) e Literatura Latino-Americana e do Caribe em Ciências da Saúde (LILACS).

Combinando descritores com operadores booleanos $O R$ e $A N D$, elaborou-se a estratégia de busca dos artigos. Nas bases LILACS/BDENF, a estratégia foi: (Idoso) $A N D$ (violência doméstica $O R$ exposição à violência) $A N D$ (envelhecimento $O R$ autonomia); no MEDLINE/PubMed ${ }^{\circledast}$, foi: ((“elder abuse”) $O R$ ("domestic violence") $O R$ (violence)) AND (("exposure to violence") $O R$ aging)) AND ((elderly) OR ("older person") $O R$ (aged) $O R$ (old)); na CINAHL, foi: ("elder abuse" OR "domestic violence" OR violence) $A N D$ ("exposure to violence" $O R$ aging) $A N D$ (elderly OR "older person" OR aged OR old); e na SciELO foi: (Idoso) $A N D$ (violência doméstica OR exposição à violência) $A N D$ (envelhecimento $\mathrm{OR}$ autonomia), (Idoso) $A N D$ (violência doméstica $O R$ exposição à violência) $A N D$ (envelhecimento $\mathrm{OR}$ autonomia) AND la: AND year_cluster:("2019" OR "2018" OR "2017" OR "2016" OR "2015") AND is_citable:("is_true") AND type:("research-article").

Os critérios de seleção dos estudos foram: artigos de abordagem quantitativa, por serem os mais amplamente difundidos em estudos com população sob risco (prevalência) e permitirem investigar as- sociaçóes entre o fenômeno em estudo e fatores de risco; artigos disponíveis na íntegra; corte temporal de 2015 a 2019, em função do Plano de Ação para o Enfrentamento da Violência Contra a Pessoa Idosa completar 15 anos no Brasil e publicaçóes nos idiomas português, espanhol e inglês. Foram excluídas revisões integrativas, conceituais e sistemáticas, bem como monografias, dissertaçóes, teses e estudos qualitativos. Para refinamento dos artigos, utilizaram-se quatro fases do diagrama Preferred Reporting Items for Systematic Reviewand MetaAnalysis (PRISMA) de fluxo de seleção de artigos. O Nível de Evidência seguiu a classificação proposta por Melnyk e Fineout-Overholt. ${ }^{(1)}$

Com a definição da amostra, elaborou-se um banco de dados. Após a primeira seleção, realizou-se a leitura para avaliação crítica e interpretação dos resultados, que foram agrupados e categorizados. Os resultados foram analisados e interpretados de forma descritiva.

\section{Resultados}

Foram identificadas 1.733 publicaçóes, das quais 1.664 foram excluídas após aplicação dos filtros e dos critérios de inclusão e exclusão, restando 69 artigos. Posteriormente, realizou-se a seleção mediante leitura na íntegra dos artigos considerados potencialmente elegíveis, cujos títulos e resumos informavam evidências sobre a violência contra pessoas idosas, totalizando 17 artigos. ${ }^{(12-28)}$ Todos os artigos da base CINAHL encontravam-se em duplicidade com outras bases de dados e, por isso, foram excluídos (Figura 1).

Os artigos foram publicados, predominantemente, nos anos de $2018(41,2 \%)^{(14-18)}$ e 2017 (23,5\%), (21-24) sendo a maioria das publicaçôes no idioma português. Dos 17 estudos, $11(64,7 \%)^{(12-17,20-22,26)}$ foram realizados na América Latina (Brasil, Equador, Colômbia e México). Os periódicos que mais contribuíram foram os da área médica $(47,1 \%)$, seguida de enfermagem (23,5\%) (Quadro 2).

Constatou-se que $11,8 \%{ }^{(18,19)}$ dos estudos expressaram Nivel de Evidência 2 e descreveram desfechos que apontam para a importância do apoio social, da 


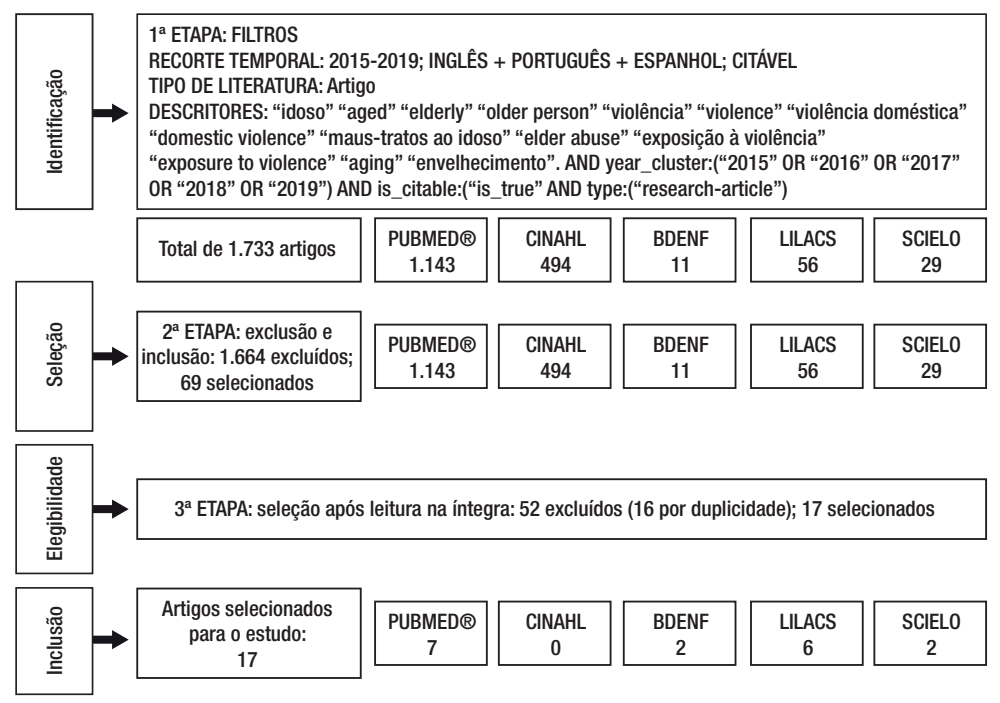

CINAHL - Cumulative Index to Nursing and Allied Health Literature; BDENF - Banco de Dados em Enfermagem; LILACS - Literatura Latino-Americana e do Caribe em Ciências da Saúde; SciELO - Scientific Electronic Library Online

Figura 1. Fluxograma do processo de seleção dos estudos

Quadro 2. Características dos artigos selecionados

\begin{tabular}{|c|c|c|c|c|c|}
\hline Ano & Título do Estudo & Periódico & Origem do Estudo & Autores & Base de Dados \\
\hline 2019 & A1: Violência sobre a pessoa idosa: um estudo documental & Revista Rene Online & Brasil & Alarcon et al. ${ }^{(12)}$ & BDENF \\
\hline 2019 & A2: A ocorrência da violência em idosos e seus fatores associados & Revista Brasileira de Enfermagem & Brasil & Maia et al..$^{(13)}$ & LILACS \\
\hline 2018 & $\begin{array}{l}\text { A3: Funcionalidad familiar y autoestima del adulto mayor, en situación de } \\
\text { maltrato. Un estudio participativo comunitário }\end{array}$ & Revista Lasallista de Investigatión & Equador & $\begin{array}{l}\text { Chiriboga et } \\
\text { al. }{ }^{(14)}\end{array}$ & SciELO \\
\hline 2018 & $\begin{array}{l}\text { A4: Violência familiar contra a pessoa idosa: expressões do fenômeno e } \\
\text { perspectivas para o seu enfrentamento }\end{array}$ & Textos \& Contextos & Brasil & Diel et al. ${ }^{(15)}$ & SCIELO \\
\hline 2018 & A5: Violência contra a pessoa idosa & Revista de Enfermagem UFPE Online & Brasil & Lopes et al. ${ }^{(16)}$ & BDENF \\
\hline 2018 & $\begin{array}{l}\text { A6: Prevalência e fatores associados à violência contra idosos cometida por } \\
\text { pessoas desconhecidas, Brasil, } 2013\end{array}$ & Epidemiologia e Serviços de Saúde & Brasil & $\begin{array}{l}\text { Alencar Júnior } \\
\text { et al. }{ }^{(17)}\end{array}$ & LILACS \\
\hline 2018 & $\begin{array}{l}\text { A7: Design and implementation of an empowerment model to prevent elder } \\
\text { abuse: a randomized controlled trial }\end{array}$ & Clinical Interventions in Aging & Irã & $\begin{array}{l}\text { Estebsari et } \\
\text { al. } .^{(18)}\end{array}$ & PubMed@ \\
\hline 2018 & $\begin{array}{l}\text { A8: Feasibility of intervention in elder self-neglecters: Setting the stage for future } \\
\text { research }\end{array}$ & Journal of Elder Abuse \& Neglect & Estados Unidos & Lee et $\mathrm{al}^{(19)}$ & PubMed® \\
\hline 2018 & A9: Frailty and life course violence: The international mobility in aging study & Archives of Gerontology and Geriatrics & $\begin{array}{l}\text { Albânia, Brasil, Canadá } \\
\text { e Colômbia }\end{array}$ & Gomes et al. (20) & PubMed@ \\
\hline 2017 & $\begin{array}{l}\text { A10: Collective violence and the health of the elderly: a cross-sectional analysis } \\
\text { of a population-based national survey in Mexico }\end{array}$ & $\begin{array}{l}\text { Revista Panamericana de Salud } \\
\text { Pública }\end{array}$ & México & $\begin{array}{l}\text { García-Peña et } \\
\text { al. }{ }^{(21)}\end{array}$ & SciELO \\
\hline 2017 & $\begin{array}{l}\text { A11: Cross-sectional study on the association between frailty and violence } \\
\text { against community-dwelling elderly people in Brazil }\end{array}$ & São Paulo Medical Journal & Brasil & Belisário et al.(22) & PubMed@ \\
\hline 2017 & $\begin{array}{l}\text { A12: Associations between the differential definitions of elder mistreatment } \\
\text { and suicidal ideation outcomes in U.S. Chinese older adults: do the definitions } \\
\text { matter? }\end{array}$ & $\begin{array}{l}\text { Journals of Gerontology. Series A, } \\
\text { Biological Sciences and Medical } \\
\text { Sciences }\end{array}$ & Estados Unidos & Dong ${ }^{(23)}$ & PubMed® \\
\hline 2017 & A13: Elder abuse within the family environment in the Azores Islands & $\begin{array}{l}\text { Revista Latino-Americana de } \\
\text { Enfermagem }\end{array}$ & Portugal & $\begin{array}{l}\text { Carmona-Torres } \\
\text { et al. }{ }^{(24)}\end{array}$ & LILACS \\
\hline 2016 & $\begin{array}{l}\text { A14: The prevalence of resident-to-resident elder mistreatment in nursing } \\
\text { homes }\end{array}$ & Annals of Internal Medicine & Estados Unidos & Lachs et al. ${ }^{(25)}$ & PubMed® \\
\hline 2016 & $\begin{array}{l}\text { A15: Prevalência de violência contra idosos e fatores associados, estudo de } \\
\text { base populacional em Florianópolis, SC }\end{array}$ & $\begin{array}{l}\text { Revista Brasileira de Geriatria e } \\
\text { Gerontologia }\end{array}$ & Brasil & Bolsoni et al. ${ }^{(26)}$ & LILACS \\
\hline 2015 & $\begin{array}{l}\text { A16: Estudo sobre pessoas idosas vítimas de violência em Portugal: sociografia } \\
\text { da ocorrência }\end{array}$ & Cadernos de Saúde Pública & Portugal & Gil et al. ${ }^{(27)}$ & LILACS \\
\hline 2015 & A17: Physical abuse against elderly persons in institutional settings & $\begin{array}{l}\text { Journal of Forensic and Legal } \\
\text { Medicine }\end{array}$ & Portugal & Frazão et al. ${ }^{(28)}$ & PubMed® \\
\hline
\end{tabular}

autoeficácia e de comportamentos promotores de saúde na redução do risco de violência/abuso e da autonegligência de idosos; 88,2\% apresentaram Nível de Evidência 4 e destacaram a prevalência e a tipologia da violência, o perfil do idoso vitimizado e fatores de risco para a violência (Quadro 3). ${ }^{(12-17,20-28)}$ 
Quadro 3. Síntese dos artigos selecionados para o estudo

\begin{tabular}{|c|c|c|c|}
\hline Estudo & Desenho do estudo/NE & Objetivo & Resultados e conclusões/desfecho \\
\hline$A 1^{(12)}$ & $\begin{array}{l}\text { Transversal, analítico e retrospectivo } \\
\text { (NE4) }\end{array}$ & $\begin{array}{l}\text { Associar as características sociodemográficas de idosos } \\
\text { vítimas de violência e dos agressores com os tipos de } \\
\text { violência }\end{array}$ & $\begin{array}{l}\text { Associação entre tipo de violência e sexo, estado conjugal, escolaridade, idade, } \\
\text { local de ocorrência e forma de notificação da vítima; predomínio da violência } \\
\text { financeira em homens e psicológica/moral em mulheres }\end{array}$ \\
\hline$A 2^{(13)}$ & $\begin{array}{l}\text { Transversal, por inquérito } \\
\text { populacional (NE4) }\end{array}$ & $\begin{array}{l}\text { Analisar ocorrência da violência em idosos e fatores } \\
\text { associados }\end{array}$ & $\begin{array}{l}\text { Formas de violência: pouco acesso a direitos sociais, violência verbal, moral/ } \\
\text { psicológica, falta de cuidados. As mulheres são as vitimas mais frequentes; } \\
\text { associação com sintomas depressivos }\end{array}$ \\
\hline$A 3^{(14)}$ & $\begin{array}{l}\text { Quantitativo, escopo de correlação e } \\
\text { corte transversal (NE4) }\end{array}$ & $\begin{array}{l}\text { Determinar a correlação entre o funcionamento familiar e } \\
\text { autoestima de adultos e idosos em situações de abuso }\end{array}$ & $\begin{array}{l}\text { Constatou-se correlação entre autoestima, funcionalidade familiar e situações de } \\
\text { abuso }\end{array}$ \\
\hline$A 4^{(15)}$ & $\begin{array}{l}\text { Descritivo, quantitativo e documental } \\
\text { (NE4) }\end{array}$ & $\begin{array}{l}\text { Analisar expressões da violência familiar contra a pessoa } \\
\text { idosa e as perspectivas de seu enfrentamento }\end{array}$ & $\begin{array}{l}\text { Principais vítimas: mulheres idosas, viúvas, com relativa autonomia econômica, } \\
\text { dependente de cuidados de saúde pela família }\end{array}$ \\
\hline$A 5^{(16)}$ & $\begin{array}{l}\text { Quantitativo, epidemiológico e } \\
\text { transversal (NE4) }\end{array}$ & Avaliar ocorrência da violência sofrida pela pessoa idosa & $\begin{array}{l}\text { Prevalência de violência física, por lesões não autoprovocadas, praticadas por } \\
\text { filhos e cônjuges }\end{array}$ \\
\hline$A 6^{(17)}$ & $\begin{array}{l}\text { Quantitativo e transversal } \\
\text { (NE4) }\end{array}$ & $\begin{array}{l}\text { Analisar associação entre característica sociodemográficas, } \\
\text { comportamentais e de saúde; e prevalência de violelncia } \\
\text { cometida por desconhecido }\end{array}$ & $\begin{array}{l}\text { Características sociodemográficas estiveram associadas à violência contra idosos } \\
\text { cometida por pessoas desconhecidas }\end{array}$ \\
\hline$A 7^{(18)}$ & Controlado e randomizado (NE2) & $\begin{array}{l}\text { Projetar e implementar uma intervenção educacional de } \\
\text { empoderamento para evitar o abuso de idosos }\end{array}$ & $\begin{array}{l}\text { Apoio social, autoeficácia e comportamentos promotores de saúde são } \\
\text { determinantes na diminuição do risco de abuso de idosos }\end{array}$ \\
\hline$A 8^{(19)}$ & $\begin{array}{l}\text { saio clínico randomizado } \\
\text { ntrolado (NE2) }\end{array}$ & $\begin{array}{l}\text { Realizar intervenções clínicas para melhorar situações } \\
\text { críticas de idosos que negligenciam a si mesmos }\end{array}$ & $\begin{array}{l}\text { A intervenção clínica possibilitou melhorar a participação de idosos em programas } \\
\text { de prevenção à autonegligência }\end{array}$ \\
\hline$A 9^{201}$ & Quantitativo e transversal (NE4) & $\begin{array}{l}\text { Estimar a prevalência de fragilidade; examinar associações } \\
\text { entre violência doméstica e fragilidade ao longo da vida }\end{array}$ & $\begin{array}{l}\text { A prevalência de fragilidade e risco para maus-tratos variou entre as cidades } \\
\text { estudadas, sendo mais baixa no Canadá e mais alta no Brasil. Abuso físico na } \\
\text { infância e exposição à violência psicológica pelo parceiro estiveram associados } \\
\text { à depressão }\end{array}$ \\
\hline$A 10^{(21)}$ & Quantitativo e transversal (NE4) & $\begin{array}{l}\text { Descrever a associação entre violência coletiva e saúde de } \\
\text { idosos no México }\end{array}$ & $\begin{array}{l}\text { A violência coletiva exerce uma influência indireta na saúde da população, } \\
\text { principalmente de idosos }\end{array}$ \\
\hline$A 11^{(22)}$ & $\begin{array}{l}\text { Transversal, analítico e observacional } \\
\text { (NE4) }\end{array}$ & $\begin{array}{l}\text { Verificar a associação entre violência contra idosos e } \\
\text { síndrome da fragilidade }\end{array}$ & $\begin{array}{l}\text { Associação entre renda e agressão física; morar com outra pessoa e dependência } \\
\text { para AIVD com agressão física e verbal; doenças com agressão física e/ou verbal }\end{array}$ \\
\hline$A 12^{(23)}$ & Quantitativo e epidemiológico(NE4) & $\begin{array}{l}\text { Explorar a associação entre maus-tratos e ideação suicida } \\
\text { em idosos chineses residentes em Chicago }\end{array}$ & $\begin{array}{l}\text { Maus-tratos, em especial, o psicológico, abuso financeiro e negligência estiveram } \\
\text { associados à ideação suicida }\end{array}$ \\
\hline$A 13^{(24)}$ & Descritivo e transversal (NE4) & $\begin{array}{l}\text { Dimensionar o abuso contra idosos vulneráveis no ambiente } \\
\text { familiar e comunitário }\end{array}$ & $\begin{array}{l}\text { Prevalência da violência psicológica; filhos como principais agressores; ser mulher } \\
\text { e pertencer a uma família disfuncional têm maior probabilidade de maus-tratos }\end{array}$ \\
\hline$A 14^{(25)}$ & Observacional e de prevalência (NE4) & $\begin{array}{l}\text { Estimar a prevalência de maus-tratos físico, verbal e sexual } \\
\text { em residentes de casas de repouso }\end{array}$ & $\begin{array}{l}\text { Prevalência de 20,2\%; violência verbal, física e sexual. Fatores associados: } \\
\text { comprometimento cognitivo e idoso institucionalizado }\end{array}$ \\
\hline$A 15^{(26)}$ & Transversal e populacional (NE4) & $\begin{array}{l}\text { Estimar prevalência de violência contra idosos e associação } \\
\text { com fatores demográficos, socioeconômicos e de saúde }\end{array}$ & $\begin{array}{l}\text { Prevalência de violência verbal; idosa mulher, solteira/divorciada maior chance } \\
\text { de maus-tratos; morar com filhos ou netos aumenta duas vezes a chance de } \\
\text { maus-tratos }\end{array}$ \\
\hline$A 16^{(27)}$ & $\begin{array}{l}\text { Quantitativo, descritivo e transversal } \\
\text { (NE4) }\end{array}$ & $\begin{array}{l}\text { Caracterizar a estrutura sociodemográfica, econômica e } \\
\text { condições de violência no contexto familiar do idoso }\end{array}$ & $\begin{array}{l}\text { Prevalência da violência física e psicológica. As vítimas eram mulheres, com idade } \\
\text { de } 70,7 \text { anos e seus agressores eram familiares }\end{array}$ \\
\hline$A 17^{(28)}$ & Quantitativo e retrospectivo (NE4) & $\begin{array}{l}\text { Proporcionar conhecimento sobre abuso físico contra idosos } \\
\text { institucionalizados e detecção correta do diagnóstico forense }\end{array}$ & $\begin{array}{l}\text { Prevalência em mulheres } \geq 75 \text { anos, com incapacidade grave e dificuldade de } \\
\text { comunicaçãao; associaçã̃o entre negligência e incapacidade }\end{array}$ \\
\hline
\end{tabular}

NE - Nivel de Evidência; BDENF - Banco de Dados em Enfermagem; LLACS - Literatura Latino-Americana e do Caribe em Ciências da Saúde; SciELO - Scientific Electronic Library Online; AIVD - Atividades Instrumentais de Vida Diária

Os artigos foram separados e compuseram duas categorias para discussão: Prevalência, tipologia e perfil da pessoa idosa vítima de violência e Fatores contribuintes e de risco para a violência contra a pessoa idosa.

\section{Discussão}

As limitaçóes deste estudo estáo relacionadas às diferentes definições das tipologias da violência, idade das populaçóes-alvo, que variam entre países desenvolvidos (65 anos) e em desenvolvimento (60 anos), resultando em dados divergentes de prevalências e na utilização somente de descritores controlados nas plataformas de busca.

\section{Prevalência, tipologia e perfil da pessoa idosa vítima de violência}

Os estudos indicaram variação dos percentuais da violência contra a pessoa idosa, entre $1,6 \%{ }^{(17)}$ a $20,2 \%,{ }^{(25)}$ cometida, na maioria das vezes, por familiares (filhos, cônjuge, netos e enteados) $)^{(15,16,20,24,27)} \mathrm{e}$, em menor grau, por desconhecidos. ${ }^{(17,25)}$

Em estudo multicêntrico realizado na Albânia, no Brasil, no Canadá e na Colômbia, encontrou-se prevalência de fragilidade e risco para violência mais alta em mulheres idosas brasileiras e mais baixa em canadenses. ${ }^{(20)}$ As evidências apontaram para o fato de que pessoas idosas que moram sozinhas ou com algum familiar apresentaram chance duas vezes maior de violência se comparadas a idosos que residiam em Instituiçóes de Longa Permanência ou que eram assistidos por 
cuidadores formais. ${ }^{(17,20,26)}$ Os tipos de violência que acometeram pessoas idosas de países de renda alta, média e baixa foram semelhantes. A violência psicológica ocupou o índice mais alto das ocorrências, seguida da

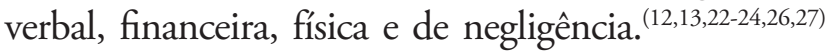
A violência sexual e a autonegligência apareceram em apenas três estudos. ${ }^{(17,19,27)}$

Houve predomínio da violência financeira em homens e da psicológica em mulheres. ${ }^{(12-14,19,20,23,27)}$ Em um estudo, ${ }^{(5)}$ as violências psicológica, física e financeira apresentaram os maiores índices, sendo que a violência sexual não foi destacada, e a autonegligência/o autoabandono foi superior à negligência - fato inusitado, uma vez que a negligência quase sempre aparece entre as primeiras ocorrências nas pesquisas. ${ }^{(12,13,22-24,26,27)}$

Os desfechos mostraram que as vítimas foram mulheres idosas, solteiras/viúvas/divorciadas, com idade avançada, menos escolarizadas, com dependência moderada/grave para realizar suas Atividades da Vida Diária, com sintomas depressivos, comprometimento cognitivo e percepção ruim de sua saúde. ${ }^{(12,15,17-19,21,22,25,26,28)} \mathrm{O}$ perfil da vítima não mudou ao longo do tempo e do lugar em que os estudos aconteceram. ${ }^{(29)}$

\section{Fatores contribuintes e de risco para a violência contra a pessoa idosa}

A idade avançada representou um dos mais importantes fatores de risco, estando relacionada ao grau de dependência e à perda da autonomia da pessoa idosa. É quando se instalam as condiçôes de fragilidade e necessidade de cuidados, em decorrência de doenças, incapacidade cognitiva, alteraçóes no sono, comprometimento cognitivo e motor, gerando aumento na demanda de cuidados para a família, quase sempre despreparada para essa nova realidade. $^{(12,15,26)}$

A disfuncionalidade familiar foi o segundo maior preditor de violência nesta pesquisa. Pertencer a uma família com disfunção suave ou severa representa probabilidade 8,35 vezes maior de sofrer maus-tratos; ${ }^{(14,23)}$ e os longevos, dependentes física e economicamente, omitem-se mais em denunciar seu agressor, pelas dificuldades de fazê-lo e por temerem perder o apoio ao denunciarem a violência. ${ }^{(12,13,15,17,20,24,26)}$
A falta de acesso a direitos sociais aparece como importante fator e gerador de violência, indicando que os dispositivos de proteção social existentes ainda parecem ser insuficientes para o enfrentamento da questão. ${ }^{(13,26)}$ No Brasil, é comum a ideia de que é exclusivamente da família a tarefa de amparar seus idosos, contudo os órgãos governamentais também têm responsabilidade no desenvolvimento das políticas de proteção social a essas pessoas. ${ }^{(30-32)}$ Os efeitos da intervenção por meio de apoio social, a autoeficácia e os comportamentos promotores de saúde na diminuição do risco de abuso de idosos mostraram-se significativos, ${ }^{(18,19)}$ comprovando que estereótipos quanto ao autocuidado, construídos em torno do imaginário da pessoa envelhecida, precisam ser modificados. ${ }^{(31,33)}$

Condiçôes crônicas, incapacidade da vítima e sintomas de depressão mostraram correlação significativa com repercussóes psicológicas que geram a prática de maus-tratos. ${ }^{(12,15,20,23,27)}$ Tal situação também aparece em estudo que destaca a presença de sinais depressivos associados a idade avançada, baixa escolaridade, insônia e doenças crônicas. ${ }^{(34)}$ A violência mostrou-se forte preditor de tentativas e suicídio completo, e, como fator redutor das tentativas de suicídio e/ou suicídio decorrente de violência, está a melhoria dos serviços de acolhimento e de suporte familiar. ${ }^{(23)}$

O envelhecimento humano é um fenômeno que requer planejamento e recursos, sendo fundamental garantir direitos com políticas públicas efetivas de proteção e prevenção. ${ }^{(15,31,32)}$ Da mesma forma, não há como a família cuidar sem um suporte que permita cuidados básicos e previna, principalmente, negligência relacionada a cuidados básicos.

\section{Conclusão}

Os artigos analisados nesta pesquisa mostram evidências que ultrapassam a simples análise de fatores isolados de violência contra a pessoa idosa. Destacam-se quatro importantes fatores: idade avançada, representando o maior fator de risco para a violência, interligada à perda de autonomia e independência e à necessidade de cuidados de terceiros; 
disfuncionalidade familiar, com probabilidade elevada para maus-tratos contra o idoso; falta de acesso a direitos sociais, indicando que os dispositivos de proteção social existentes ainda são insuficientes para o enfrentamento da violência; condiçóes crônicas de incapacidade da vítima e de sintomas de depressão, com repercussões psicológicas que levam à violência e à tentativa de suicídio.

\section{Agradecimentos}

Os autores agradecem ao Programa de Mestrado Profissional em Enfermagem em Saúde Pública da Universidade do Estado do Amazonas, à Fundação de Amparo à Pesquisa do Estado do Amazonas FAPEAM e ao Programa Acadêmico em Ciências do Cuidado-PACCS da Universidade Federal Fluminense.

\section{Referências}

1. Degani GC, Mendes KD, Storti LB, Marques S. Advanced mobile prehospital nursing care for elderly people post-trauma: integrative review. Rev Bras Enferm. 2019;72 Suppl 2:274-83.

2. Souza MM, Ongaro JD, Lanes TC, Andolhe R, Kolankiewicz AC, Magnago TS. Patient safety culture in the Primary Health Care. Rev Bras Enferm. 2019;72(1):27-34

3. Poltronieri BC, Souza ER, Ribeiro AP. Violência no cuidado em instituições de longa permanência para idosos no Rio de Janeiro: percepções de gestores e profissionais. Saude Soc. 2019;28(2):21526.

4. World Health Organization (WHO). International Network for the Prevention of Elder Abuse (INPEA). Missing Voices: views of older persons on elder abuse [Internet]. Geneva: WHO; 2002. [cited 2020 May 27]. Available from: https://www.who.int/ageing/publications/ missing_voices/en/

5. Lopes ED, Ferreira AG, Pires CG, Moraes MC, D’Elboux MJ. Elder abuse in Brazil: an integrative review. Rev Bras Geriatr Gerontol. 2018;21(5):628-38.

6. Backes C, Beuter M, Venturini L, Benetti ER, Bruinsma JL, GirardonPerlini NM, et al. A prática da contenção em idosos: revisão integrativa. Acta Paul Enferm. 2019;32(5):578-83.

7. Brasil. Presidência da República. Subsecretaria de Direitos Humanos. Plano de ação para o enfrentamento da violência contra a pessoa idosa [Internet]. Brasília (DF): Subsecretaria de Direitos Humanos; 2005 [citado 2020 Mai 27]. Disponível em: https:// bvsms.saude.gov.br/bvs/publicacoes/plano_acao_enfrentamento_ violencia_idoso.pdf

8. Diniz M. Disque 100 registra 142 mil denúncias de violações em 2017 [Internet]. Brasília (DF): Agência Brasil; 2017. [citado 2019 Dez 5]. Disponível em https://agenciabrasil.ebc.com.br/direitos-humanos/ noticia/2018-04/disque-100-registra-142-mil-denuncias-deviolacoes-em-2017
9. Mendes KD, Silveira RC, Galvão CM. Revisão integrativa: método de pesquisa para a incorporação de evidências na saúde e na enfermagem. Texto Contexto Enferm. 2008;17(4):758-64.

10. Polit DF, Beck CT. Fundamentos da pesquisa em enfermagem: avaliação de evidências para a prática de enfermagem. 9th ed. Porto Alegre: ArtMed; 2018.

11. Melnyk BM, Fineout-Overholt E. Making the case for evidence-based practice. In: Melnyk BM, editor. Fineout-Overholt E. Evidence-based practice in nursing \& healthcare: a guide to best practice. Philadelphia: Lippincot Williams \& Wilkins; 2005. p. 3-24.

12. Alarcon MF, Damaceno DG, Lazarini CA, Braccialli LA, Sponchiado VB, Marin MJ. Violência sobre a pessoa idosa: um estudo documental. Rev Rene Online. 2019;20:e41450.

13. Maia PH, Ferreira EF, Melo EM, Vargas AM. Occurrence of violence in the elderly and its associated factors. Rev Bras Enferm. 2019;72 Suppl 2:64-70.

14. Viteri Chiriboga $E$, Terranova Barrezueta $A E$, Velis Aguirre LM. Funcionalidad familiar y autoestima del adulto mayor, en situación de maltrato. Un estudio participativo comunitario. Rev Lasallista Investig. 2018;15(2):300-14.

15. Diel M, Barbiani R. Violência familiar contra a pessoa idosa: expressões do fenômeno e perspectivas para o seu enfrentamento. Textos \& Contextos (Porto Alegre). 2018;17(2):379-92.

16. Lopes LG, Leal MC, Souza EF, Silva SZ, Guimarães NN, Silva LS. Violência contra a pessoa idosa. J Nurs UFPE Online. 2018;12(9):2257-68.

17. Alencar Júnior FO, Moraes JR. Prevalência e fatores associados à violência contra idosos cometida por pessoas desconhecidas, Brasil, 2013. Epidemiol Serv Saúde Online. 2018;27(2):e2017186.

18. Estebsari F, Dastoorpoor M, Mostafaei D, Khanjani N, Khalifehkandi ZR, Foroushani AR, et al. Design and implementation of an empowerment model to prevent elder abuse: a randomized controlled trial. Clin Interv Aging. 2018;13:669-79.

19. Lee JL, Burnett J, Xia R, Smith SM, Dyer CB. Feasibility of intervention in elder self-neglecters: setting the stage for future research. J Elder Abuse Negl. 2018;30(3):223-35.

20. Dos Santos Gomes C, Pirkle CM, Zunzunegui MV, Taurino Guedes D, Fernandes De Souza Barbosa J, Hwang P, et al. Frailty and life course violence: the international mobility in aging study. Arch Gerontol Geriatr. 2018;76:26-33.

21. García-Peña C, Sánchez-Garrido N, Wynne-Bannister EG, MorenoPeniche B, Pérez-Zepeda MU. Collective violence and the health of the elderly: a cross-sectional analysis of a population-based national survey in Mexico. Rev Panam Salud Publica. 2017;41:e29.

22. Belisário MS, Dias FA, Pegorari MS, Paiva MM, Ferreira PC, Corradini FA, et al. Cross-sectional study on the association between frailty and violence against community-dwelling elderly people in Brazil. Sao Paulo Med J. 2018;136(1):10-9.

23. Dong X. Associations between the differential definitions of elder mistreatment and suicidal ideation outcomes in U.S. Chinese older adults: do the definitions matter? J Gerontol A Biol Sci Med Sci. 2017;72 Suppl 1:S82-9.

24. Carmona-Torres JM, Carvalhal-Silva RM, Vieira-Mendes MH, RecioAndrade B, Goergen T, Rodríguez-Borrego MA. Elder abuse within the family environment in the Azores Islands. Rev Lat Am Enfermagem. 2017;25:e2932.

25. Lachs MS, Teresi JA, Ramirez M, van Haitsma K, Silver S, Eimicke JP, et al. The prevalence of resident-to-resident elder mistreatment in nursing homes. Ann Intern Med. 2016;165(4):229-36. 
26. Bolsoni CC, Coelho EB, Giehl MW, d’Orsi E. Prevalência de violência contra idosos e fatores associados, estudo de base populacional em Florianópolis, SC. Rev Bras Geriatr Gerontol. 2016;19(4):67182.

27. Gil AP, Santos AJ, Kislaya I, Santos C, Mascoli L, Ferreira Al, et al. [A sociography of elderly victims of family violence in Portugal]. Cad Saude Publica. 2015;31(6):1234-46.

28. Frazão SL, Correia AM, Norton P, Magalhães T. Physical abuse against elderly persons in institutional settings. J Forensic Leg Med. 2015;36:54-60.

29. Brasil. Ministério Público do Distrito Federal e Territórios (MPDFT). Mapa da violência contra a pessoa idosa no Distrito Federal: análise das denúncias recebidas entre 2008 e 2018 realizada pela Central Judicial do Idoso [Internet]. 4a ed. Brasília (DF): MPDFT; 2019 [citado 2020 Mai 27]. Disponível em: https://www.tjdft.jus. br/informacoes/cidadania/central-judicial-do-idoso/publicacoes/ mapa-da-violencia-1/mapa_violencia_pesssoa_idosa_df_4a_ edicao_2019-7.pdf

30. Moreira WC. Violence against the elderly: a public health issue. ReOnFacema. 2017;3(1):440-3.

31. Faller JW, TestonEF, Marcon SS. Estrutura conceptual do envelhecimento em diferentes etnias. Rev Gaúcha Enferm. 2018;39(0):e66144.

32. Santana 10, Coutinho MP, Vasconcelos DC, Coutinho ML. Representações sociais sobre violência urbana: um estudo no contexto do envelhecimento. Rev Kairós Gerontologia. 2015;18(4):247-68.

33. Costa IP, Bezerra VP, Pontes ML, Moreira MA, Oliveira FB, Pimenta CJ, et al. Qualidade de vida de idosos e sua relação com o trabalho. Rev Gaúcha Enferm. 2018;39:e2017-13.

34. Nery BL, Cruz KC, Faustino AM, Santos CT. Vulnerabilities, depression, and religiosity in the elderly hospitalised in an emergency unit. Rev Gaúcha Enferm. 2018;39:e20170184. 